\title{
Research Paper \\ The Effect of Intestinal Natural Micro Flora Removal on Susceptibility to Have Seizure in Male Wistar Rats
}

\author{
Saeed Tahmasebi ${ }^{1} \odot$, *Sharbanoo Oryan ${ }^{2} \odot$, Hamid Reza Mohajerani ${ }^{3} \odot$, Neda Akbari ${ }^{3},{ }^{*}$ Mohammad Reza Palizvan ${ }^{4}$
}

1. Department of Biology, Faculty of Basic Science, Science and Research Branch, Islamic Azad University, Tehran, Iran.

2. Department of Animal Biology, Faculty of Biological Science, University of Kharazmi, Tehran, Iran.

3. Department of Microbiology, Faculty of Basic Science, Arak Branch, Islamic Azad University, Arak, Iran.

4. Department of Physiology, School of Medicine, Arak University of Medical Sciences, Arak, Iran. moval on Susceptibility to Have Seizure in Male Wistar Rats (Persian)]. Journal of Arak University of Medical Sciences (JAMS). 2020; 23(3):326-337. https://doi.org/10.32598/JAMS.23.3.6110.1

https://doi.org/10.32598/JAMS.23.3.6110.1

Key words: Microflora, Probiotics, Seizure, Rat

\section{ABSTRACT}

Article Info:

Received: 07 Feb 2020

Accepted: 06 Jun 2020

Available Online: 01 Aug 2020

Background and Aim Epilepsy is a common brain disorder. Brain function can be affected by the intestinal microflora. The intestinal microflora have a major role in modulating immune responses, producing essential metabolites and mediating neurotransmitters. Antibiotics can affect and reduce these roles and functions by eliminating the intestinal microflora. The aim of this study was to investigate the effect of intestinal natural micro flora removal on seizure susceptibility and seizure behavior modification with the use of probiotics in male Wistar rats.

Methods \& Materials This study was performed on 32 male Wistar rats with weight range 200-250 gr. The animals were randomly divided into four groups: 1 . Control group; 2. Antibiotic group; 3. Probiotic group and 4. Antibiotic + probiotic group. To remove the microflora, antibiotics (neomycin, ampicillin, and metronidazole) for three weeks and for replacement of microflora, probiotics (Lactobacilli casei, Lactobacillus acidophilus and Bifidobacterium bifidum) for four weeks were administered. Seizures were performed by intraperitoneal injection of pentylentrazole. The microflora was examined by the MRS Agar medium and the Pure Plate method. The data were statistically analyzed in Graph Pad Prism V. 8.

Ethical Considerations This study was approved by the Research Ethics Committee of Arak University of Medical Sciences (Code: IR.ARAKMU.REC.1395.176).

Results The use of antibiotics lead to decrease the number of intestinal bacteria $(P<0.0001)$, increased the severity and stability of seizure stages $(P<0.05)$ and decreased the time delay of seizure onset $(P<0.05)$ compared to the control group. Probiotic consumption by modifying the intestinal microflora $(P<0.0001)$ reduced the severity of seizure and increased the time delay of seizure onset $(P<0.05)$.

Conclusion Elimination of microflora has the potential to induce seizures, which can be compensated by administration of probiotics.
* Corresponding Author:

Sharbanoo Oryan, PhD.

Address: Department of Animal Biology, Faculty of Biological Science, University of Kharazmi, Tehran, Iran.

Tel: +98 (21) 80633368

E-mail: sh-oryan@khu.ac.ir

\section{Mohammad reza Palizvan, PhD.}

Address: Department of Physiology, School of Medicine, Arak University of Medical Sciences, Arak, Iran.

Tel: +98 (86) 34173502

E-mail: dr.palizvan@arakmu.ac.ir 


\section{Extended Abstract}

\section{Introduction}

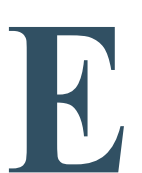

pilepsy is a common neurological disorder after a stroke. The disease is characterized by dysfunction of brain neurons, which manifests in the form of periodic and unpredictable seizure [1]. Researches have shown that brain function is affected by intestinal microbiota [6]. The natural gut flora play role by altering immune responses, producing essential metabolites and neurotransmitters [9]. These changes can subsequently effect on all pathways involved in neuronal stimulation and inflammation [10]. Elimination of the intestinal natural micro flora by the use of antibiotics will remove their beneficial effects [23-25]. The aim of this study was to investigate the effect of intestinal natural micro flora removal on seizure susceptibility and seizure behavior modification with the use of probiotics in male Wistar rats.

\section{Materials and Methods}

This study was performed on 32 male Wistar rats with weight range 200-250 gr. The mice were prepared from the Razi Institute of Tehran. Animals were maintained under controlled temperature $\left(22 \pm 2^{\circ} \mathrm{C}\right)$ and a 12:12-h light: dark cycle. The animals were randomly divided into four groups: (1) Control group, (2) Antibiotic group, (3) Probiotic group and (4) Antibiotic + probiotic group. To remove the micro flora, antibiotics (neomycin, ampicillin and metronidazole) for three weeks and for replacement of micro flora, probiotics (Lactobacilli casei, Lactobacillus acidophilus and Bifidobacterium bifidum) for four weeks were administered $[26,27]$. Seizures were performed by intraperitoneal injection of pentylene tetrazole $(45 \mathrm{mg} / \mathrm{kg})$. The time between injection of pentylene tetrazole and the start of the second also fifth stage of seizures, the length of time the animals are in stage five seizure and Maximum seizure stage, measured as an important indicator of seizure behavior [28]. The micro flora was examined by the MRS Agar medium and the Pure Plate method [27]. The data were statistically analyzed in Graph Pad Prism v. 8.

\section{Results}

The results of this study showed that the use of antibiotics lead to decrease the number of intestinal bacteria $(\mathrm{P}<0.0001)$, increased the severity and stability of seizure stages $(\mathrm{P}<0.05)$ and decreased the time delay of seizure onset $(\mathrm{P}<0.05)$ compared to the control group. Probiotic consumption by modifying the intestinal micro flora
$(\mathrm{P}<0.0001)$ reduced the severity of seizure and increased the time delay of seizure onset $(\mathrm{P}<0.05)$.

\section{Discussion}

The results of the present study showed that the use of antibiotics reduces the beneficial bacteria in the gut, including lactobacillus and bifidobacteria. Similarly, other colleagues achieved these results [26, 29]. Antibiotic treatment also increased the severity of seizures and reduced the time required for seizures to occur in the seizure model caused by pentylene tetrazole. This means that removing the natural intestinal flora can subsequently eliminate the effects of beneficial bacteria. For example, Jesus-Servando et al. showed that chronic stress can facilitate epilepsy in an animal model by altering the gut microbial profile [40]. Xie et al. reported that ketogenic diets alter intestinal microbiome in resistant epileptic children [41].

Comparison of bacterial colony counts in rat feces showed that taking probiotic supplements, a mixture of lactobacillus and bifidobacteria, could restore the population of natural intestinal flora lost due to antibiotic treatment. Allori et al. in the animal and Plummer et al. in the human model proved that taking probiotic supplements could restore the bacteria removed from the gut $[27,31]$. Comparison of seizure behavior parameters in different groups showed that the use of probiotic supplementation moderated the seizure behavior. Similar to the present study, Bagheri and her colleagues reported a reduction in pentylene tetrazole-induced Kindling attacks due to probiotic use [44].

\section{Conclusion}

It seems that elimination of micro flora has the potential to induce seizures, which can be compensated by administration of probiotics.

\section{Ethical Considerations}

\section{Compliance with ethical guidelines}

This study with Ethics Code IR.ARAKMU. REC.1395.176 was approved by the Research Ethics Committee of Arak University of Medical Sciences.

\section{Funding}

This article was supported by Islamic Azad University of Tehran, Science and Research Branch, (Code: 9412) and exteracted from the $\mathrm{PhD}$. dissertation of the first author Department of Biology, Faculty of Basic Science, Science and Research Branch, Islamic Azad University. 


\section{Authors' contributions}

All authors contributed in preparing this article.

Conflicts of interest

The authors declared no conflicts of interest.

Acknowledgements

The authors would like to thank the Islamic Azad University of Tehran, Science and Research Branch for financial support. 


\section{اثر حذف ميكروفلور طبيعى روده بر استعداد ابتلابه تشنج در موشهاى صحرايى نر نثاد ويستار}

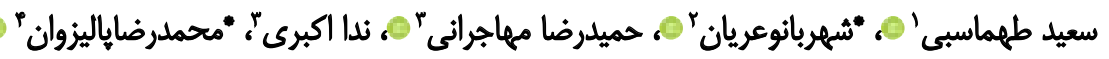

ا. كروه زيست شناسى، دانشكده علوم يايه، واحد علوم و تحقيثات، دانشكاه آزاد اسلامي، تهران، ايران.

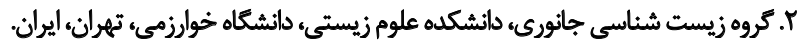

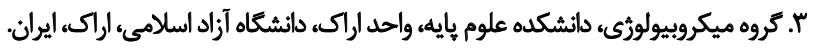

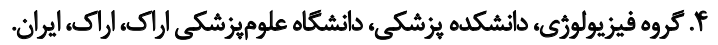

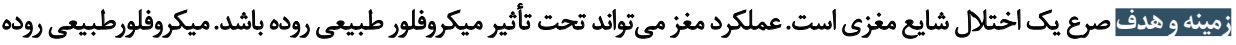

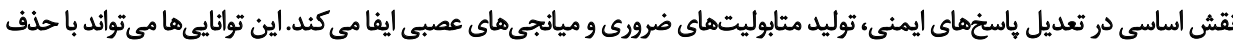

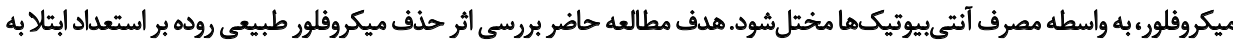

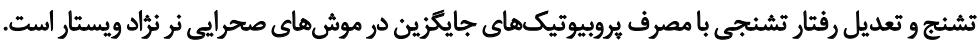

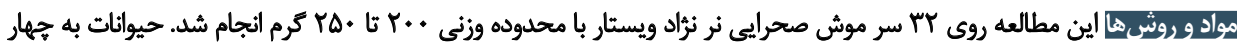

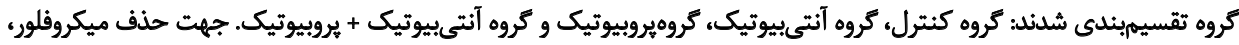

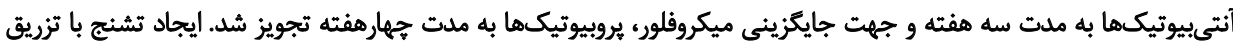

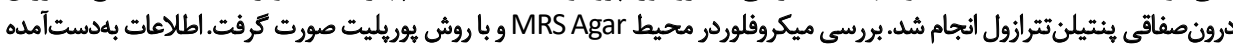

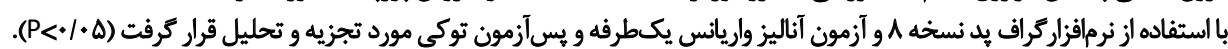

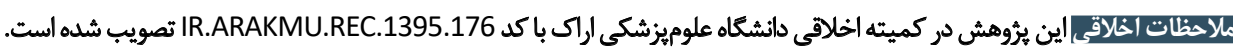

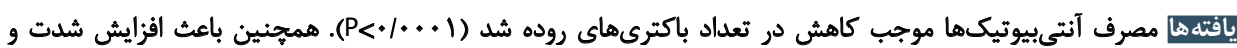

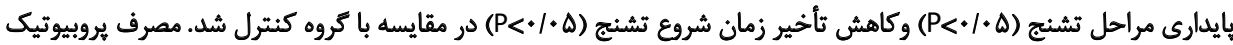

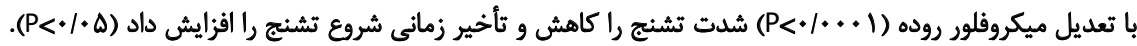

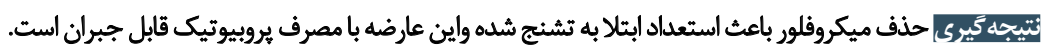

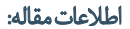

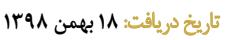

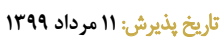

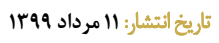

كليدوأرهها: ميكروفلور، يروبيوتيك،

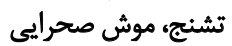

بالاى بيمارى و مشكلات ناشى از آن انجام هر مطالعهاي

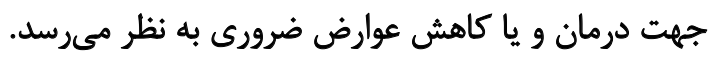

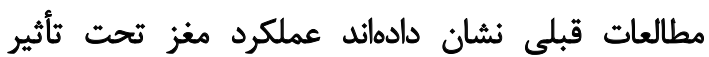

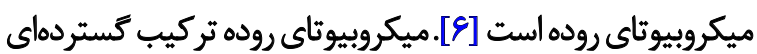

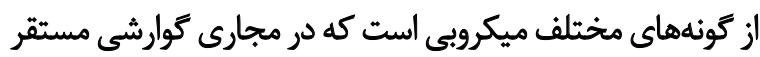

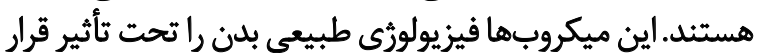

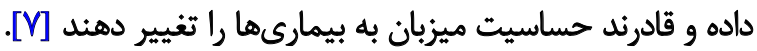

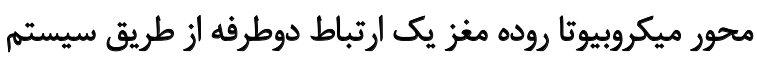

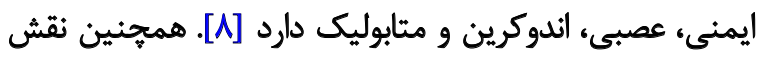

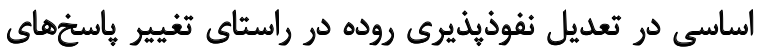

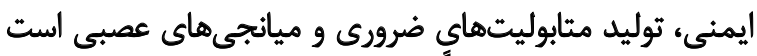

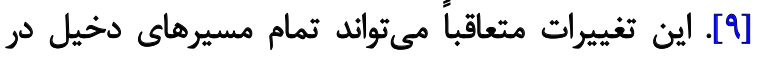

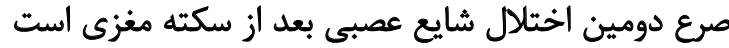

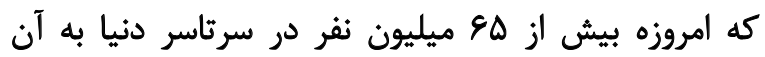

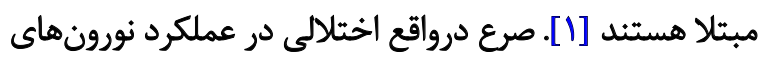

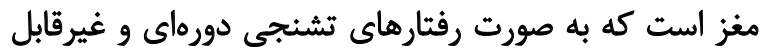

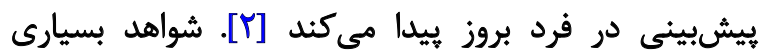

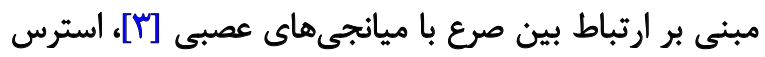

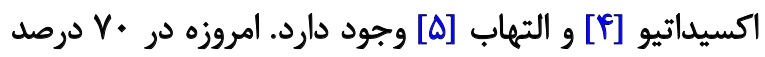

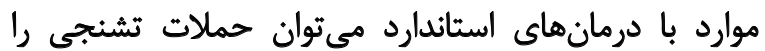

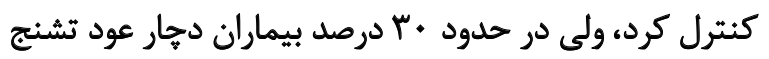

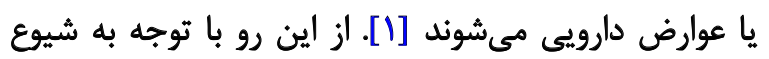

متممدرضاياليزوان

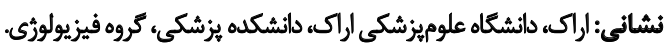

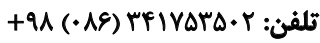
يست الكترونيكى: palizvan@arakmu.ac.ir

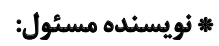

شهربانوعريان

نشانى: تهران، دانشعاه خوارزمي، دانشكده علوم زيستى، كروه زيست شئاسى جانورى.

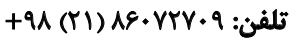
يست الكترونيكى: sh-oryan@khu.ac.ir 
بيفيدوباكتريومها) به عنوان جايكَّزين ميكروفلور طبيعى روده در موشهاى صحرايى نر نرّاد ويستار است.

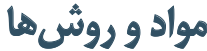

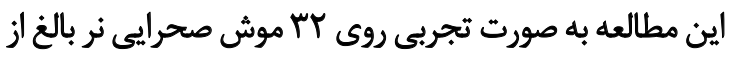

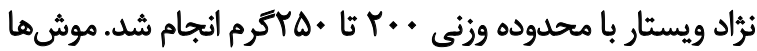

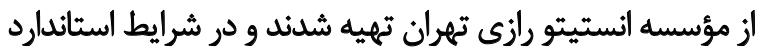

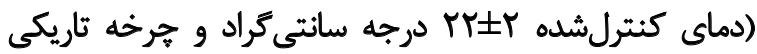

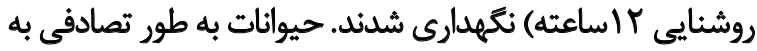

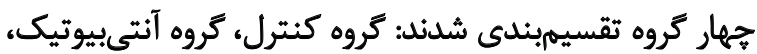

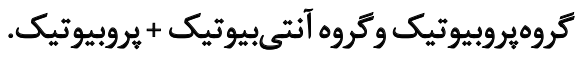

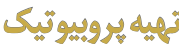

يروبيوتيكهاىمورداستفادمخلوطىازسهسويهلاكثوباسيل كازئى،

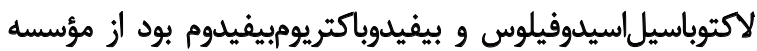
تكثرن با نام تجارى بروويتا تهيه شده بودي

L

با توجه به اهميت و نقش لاكتوباسيلها و بيفيدوباكتريومها

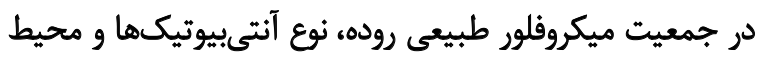

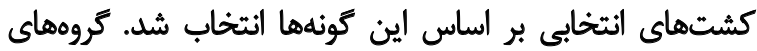

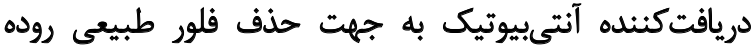

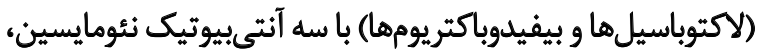

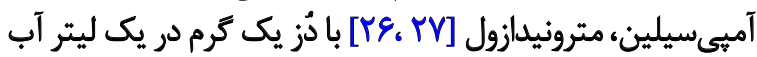

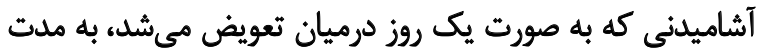

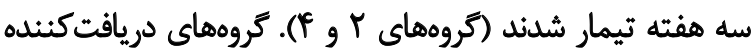

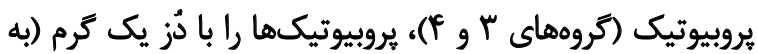

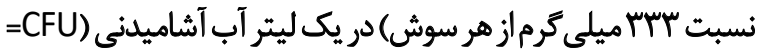

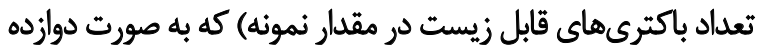

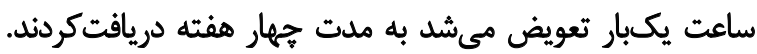

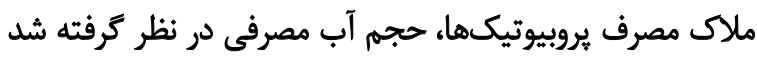

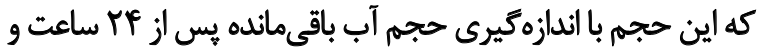

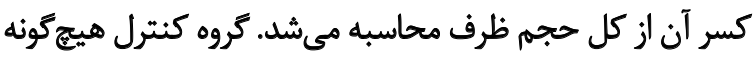

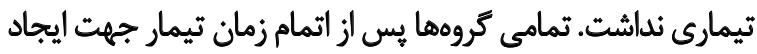

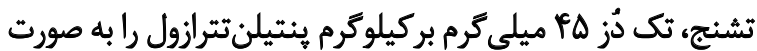
درون صفاقى دريافت كردند (تصوير شماره ()).

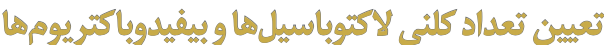

هماند مطالعات قبلى براي اطمينان از عملكرد آنتىبيوتيكها

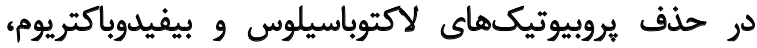

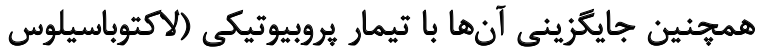

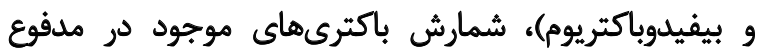

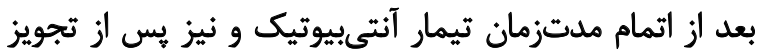

تحريكيذيرى نورونى و التهابى را تحت تأثير قرار دهد [ • [1].

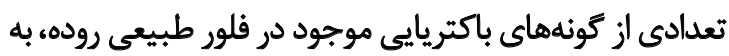

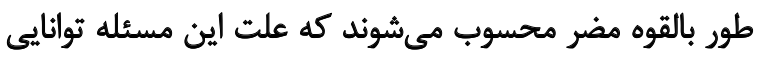

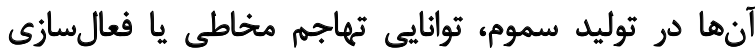

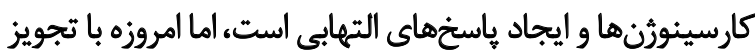

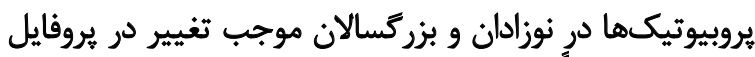

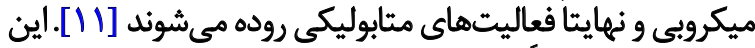

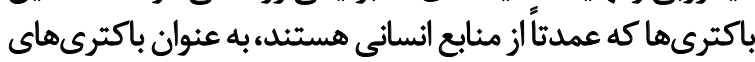

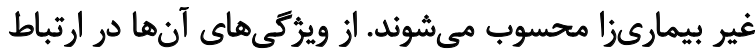

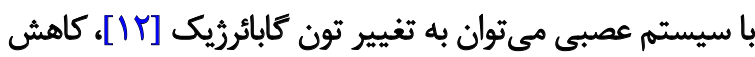

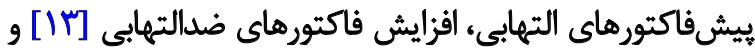
ظرفيت آنتى اكسيدانى اشاره كرد [ [ If]. اثبات شده است كه بروبيوتيكها اين عمل رااز طريق اقزايش

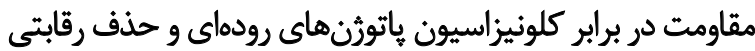

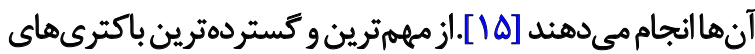

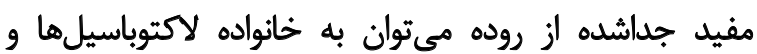

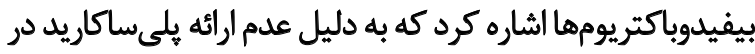

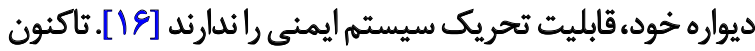

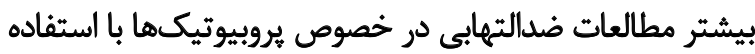

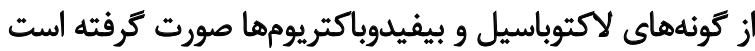

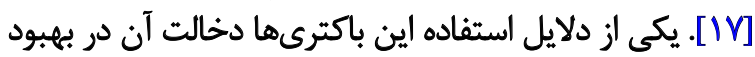

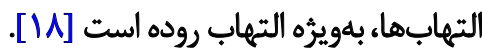

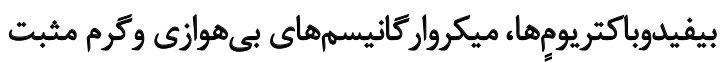

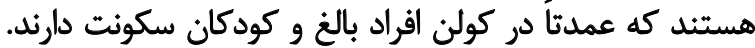

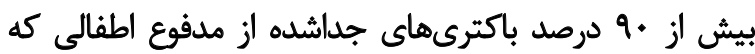

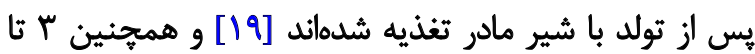

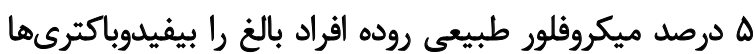

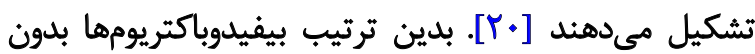

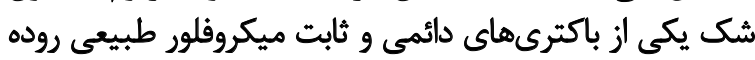

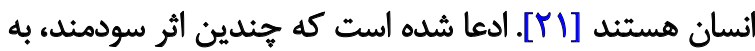

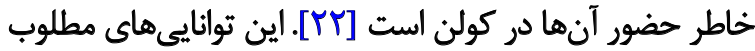

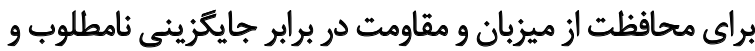

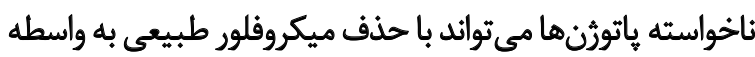

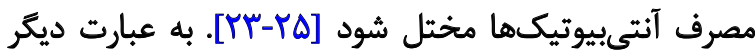

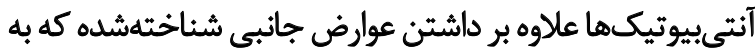

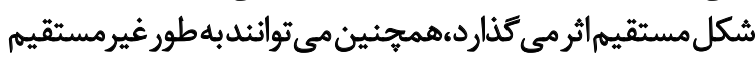

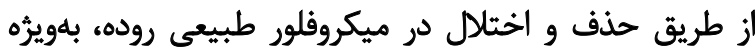

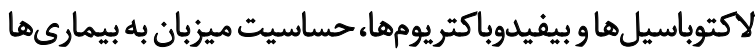

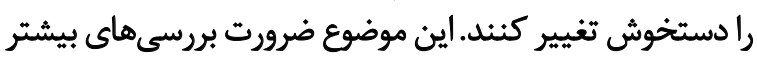

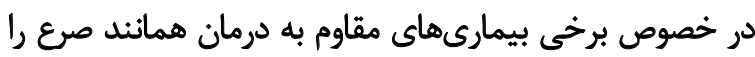

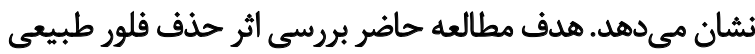

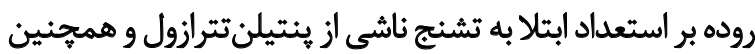

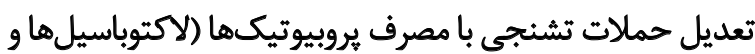




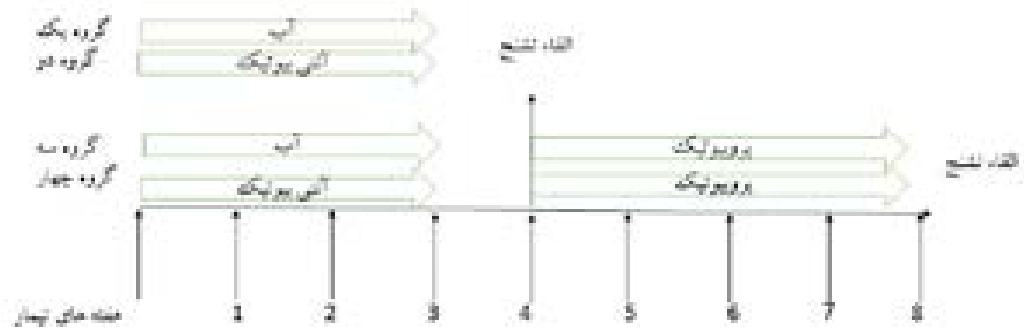

tist

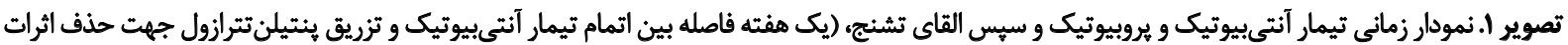

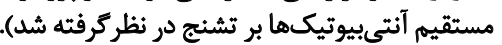

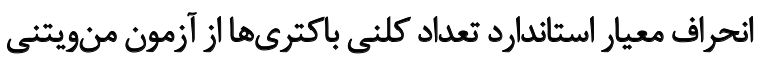

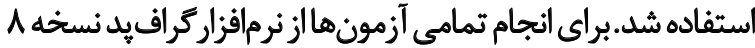

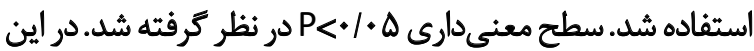

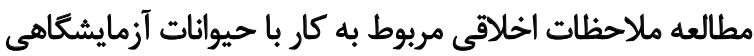
با كد اخلاق

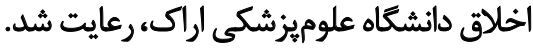

batiol

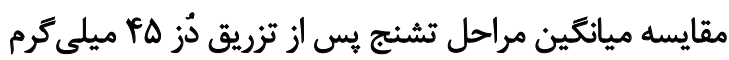

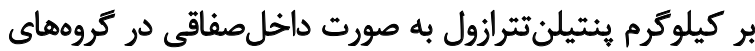
تيمارى مختلف نشان داد تيمار سههفتهاى موش هاى صني صحرايى توسط آنتىبيوتيكهاى آميىسيلين، نئومايسين و مترونيدازول در مقايسه با كروه كنترل (بدون ثيمار آنتىبيوتيك) ميانغين

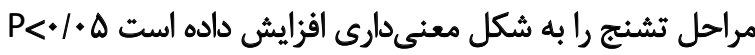
(تصوير شماره (Y).

ميانكين مراحل تشنج دريروه تيمارشده با آنتىبيوتيك كه مدئ

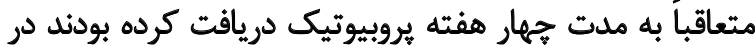

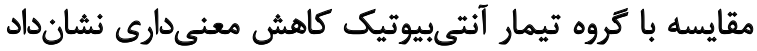

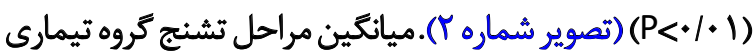

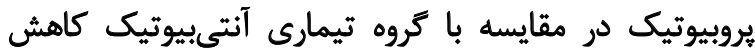

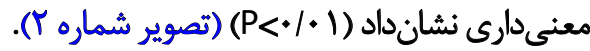
ميانكين زمان تأخير شروع مرحله دوم تشنج در كروه

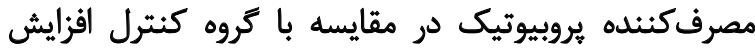

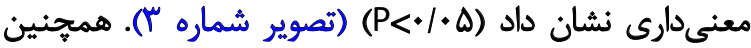

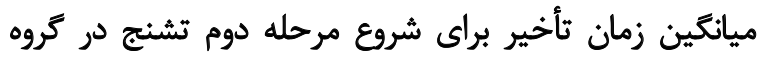

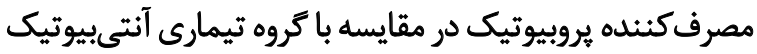

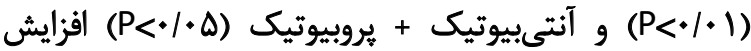

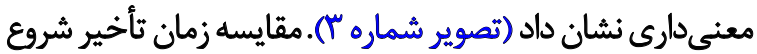

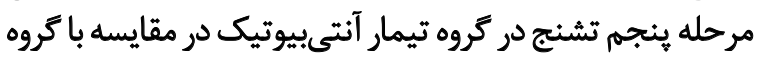

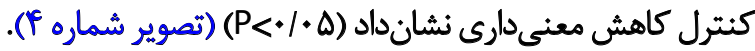

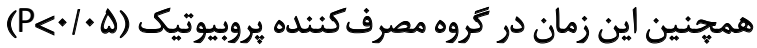

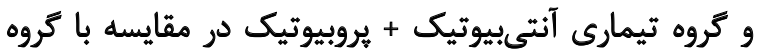

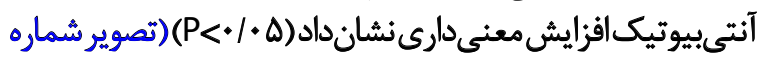

بروبيوتيك، از محيط كشت MRS Agar و روش يوريليت استفاده

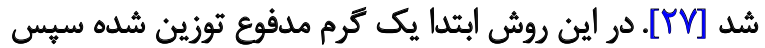

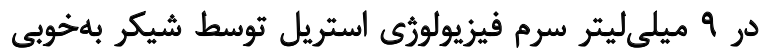

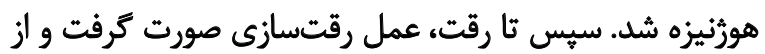

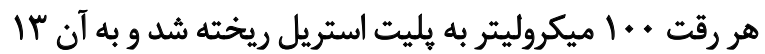

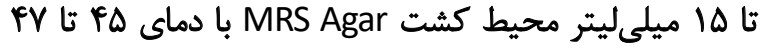

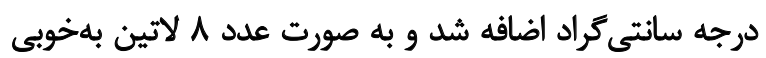

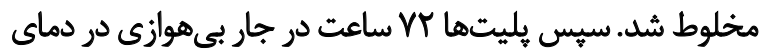

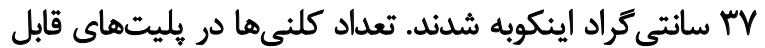

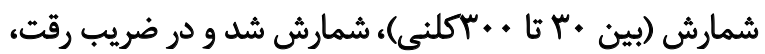

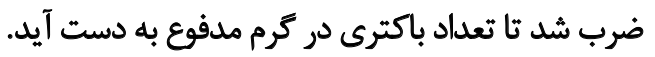

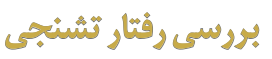

ياسخهاى تشنجى حيوان بر اساس تحقيقات قبلى به شكل زير مورد بررسي قرارئرفت[YA] مرحله صفر= عدم ياسخ، مرحله اول: انقباض عضلات صورت و

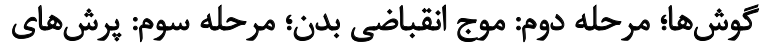

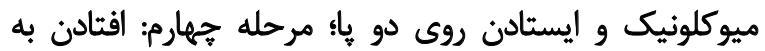

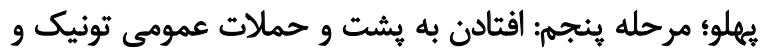

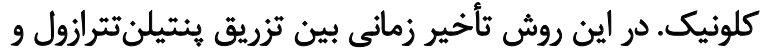

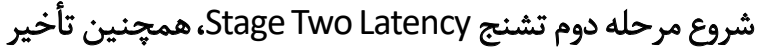

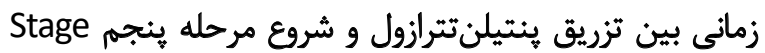
Five Latency به سر مىبرند Stage Five Duration و حداكثر مرحله تشنج Seizure Stage به عنوان شاخص مهاى مهم در رفتار تشنجى اندازميرى شد.

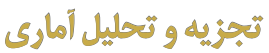

براي مقايسه ميائَين و انحراف معيار استاندارد مراحل تشنج در

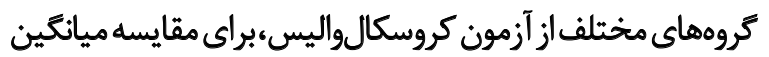

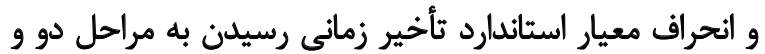

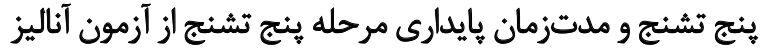
واريانس يكىطرفه و يسآزمون توكى و براى مقايسه ميانكين و ماني 

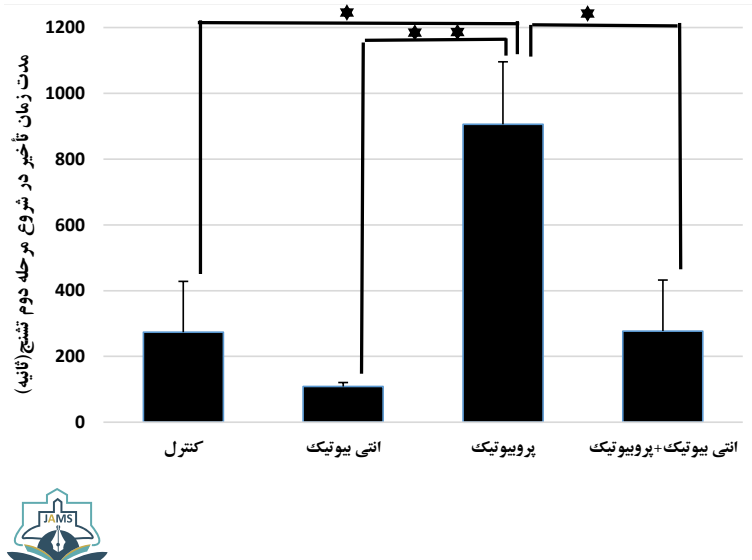

تصوير با. اثر تيمارأنتيبيوتيك و بروبيوتيك بر ثأخير شروع مرحله دو تشنتج.

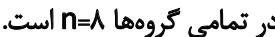

با آنتىبيوتيك كاهش معنى دارى در مراحل تشنج داشته است. مقايسه ميانكين به علاوه انحراف معيار استاندارد زمان تأخير

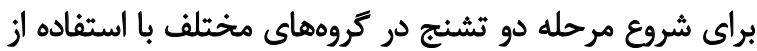

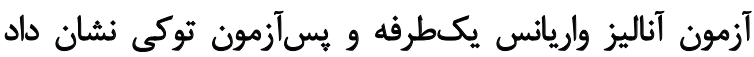

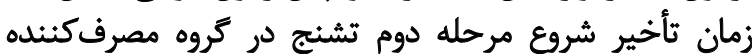

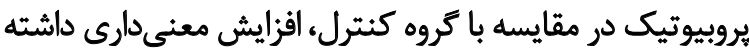

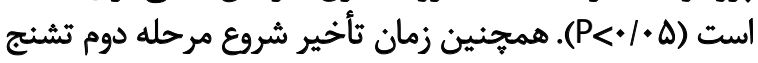

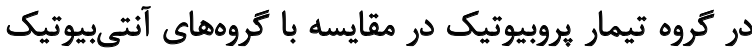

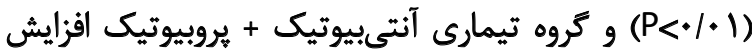

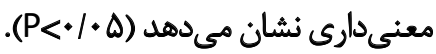

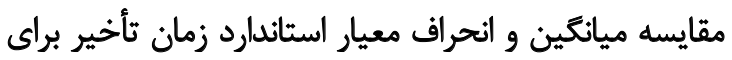

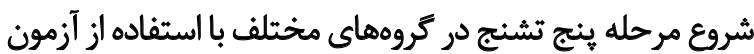

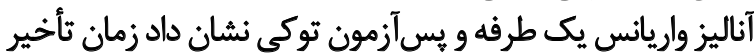

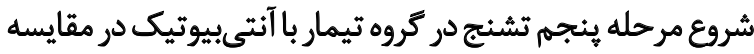

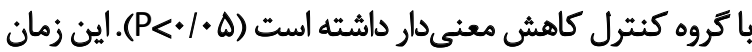

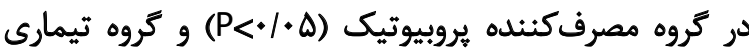

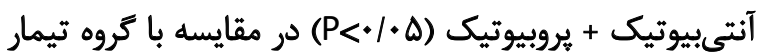
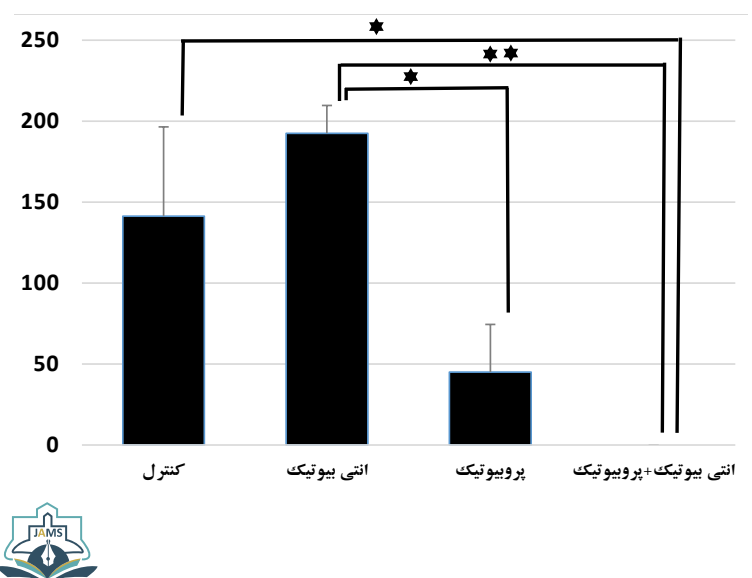

تصوير هـ اثر ثيمار آنتي بيوتيك و يروبيوتيك بر هايدارى مرحله يثج تشنتج، در

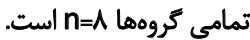

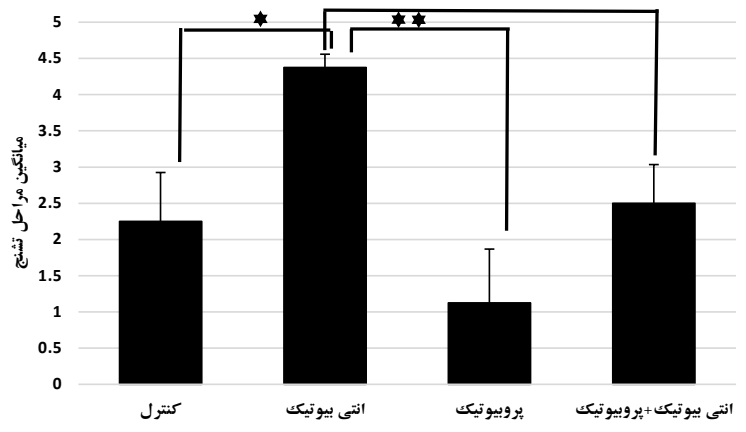

5

تصوير Y.اثر تيمار آنتىبيوتيك و بروبيوتيك بر مراحل تشُنج، در تمامى كروهها n=人

(f). ميانكين زمان غايدارى مرحله ينجم تشنج در كروه تيمارشده

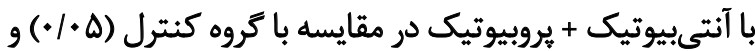

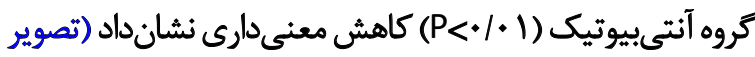

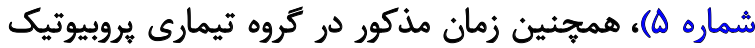

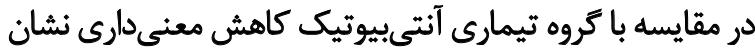

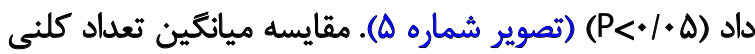

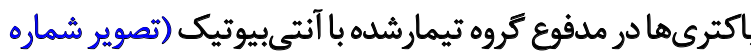

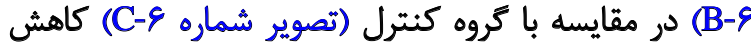

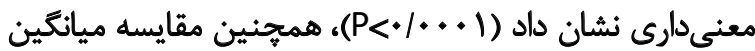

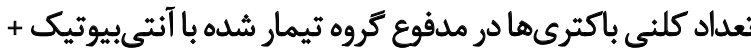

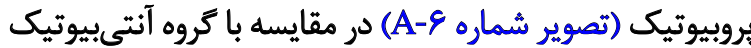

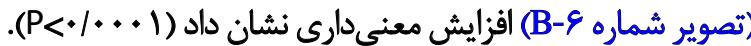

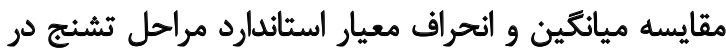

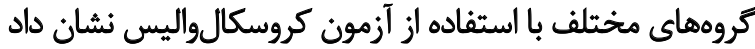

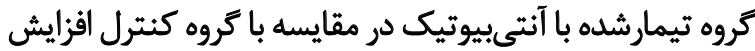

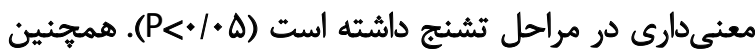

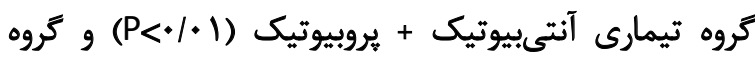

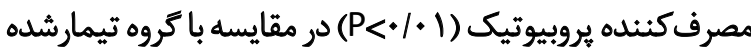
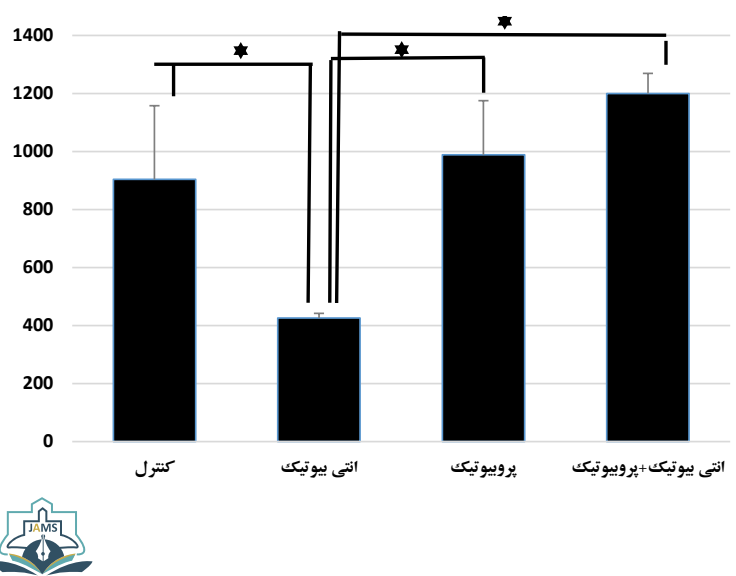

تصوير P.اثر تيمارآنتيبيوتيك و بروبيوتيك بر تأخير شروع مرحله ينج تشنج،

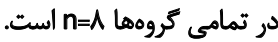




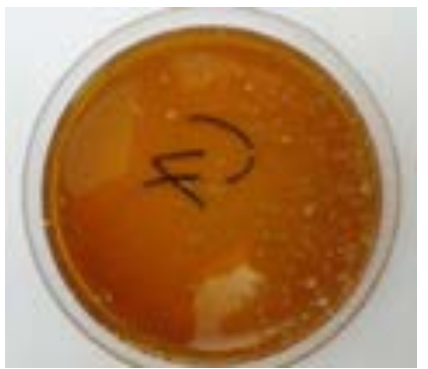

C

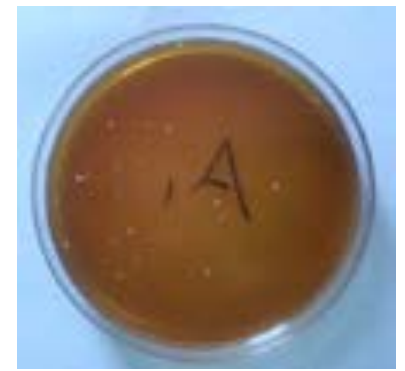

B

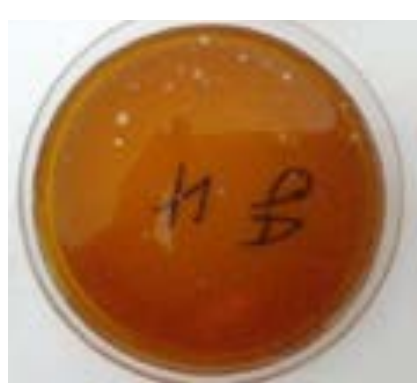

A تصوير و. اثر آنتىبيوتيك و مكمل بروبيوتيك بر فلور طبيعى روده، در تمامى تروهها n=1 است

مشاهده شد. در مقابل مصرف مكمل يروبيوتيك موجب افزايش

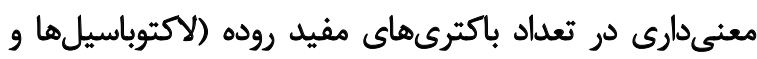

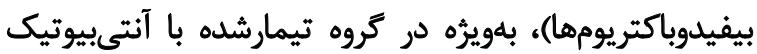

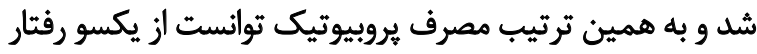

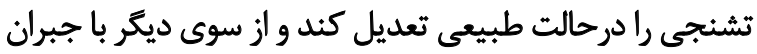

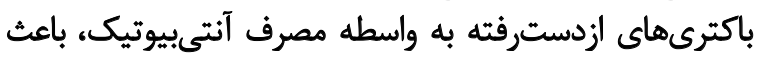

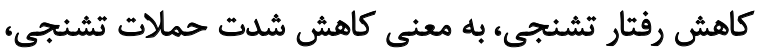

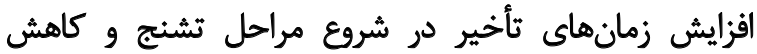
مدتزمان هايدارى تشنج شود.

بر اساس يافتههاى محققان، مصرف آنتىبيوتيكها تعادل

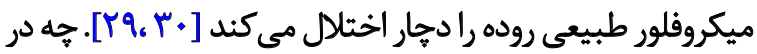

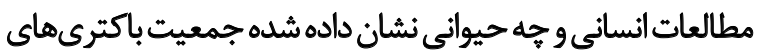

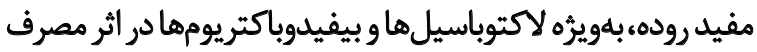

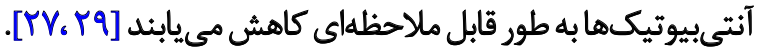

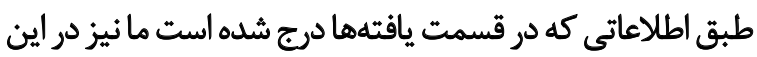

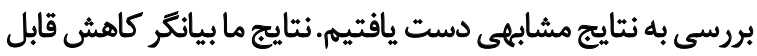

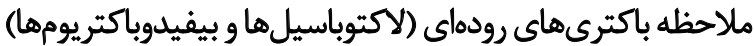

$$
\text { در اثر مصرف آنتىبيوتيكها بودي }
$$

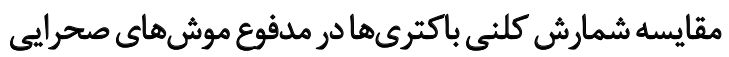

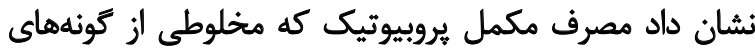

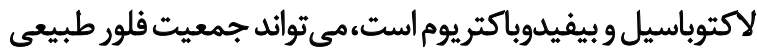

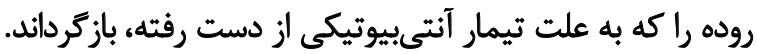

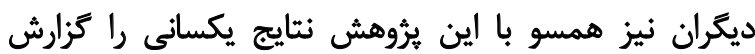

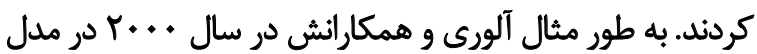

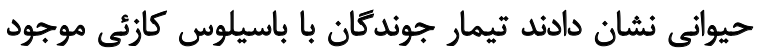

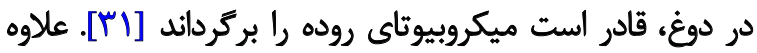

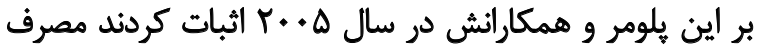

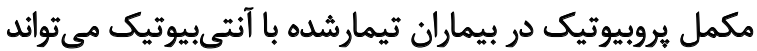

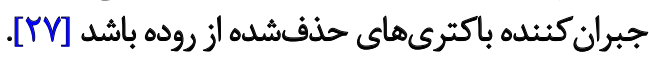

طاكنون بسيارى از مطالعات شواهدى را مبنى بر نقش فلور طبيعى روده در عملكرد سيستم عصبى مركزى ارئ ارائه كردهاند
آنتىبيوتيك افزايش معنى دارى نشان داده است.

مقايسه ميانكين و انحراف معيار استاندارد زمان بإيداري

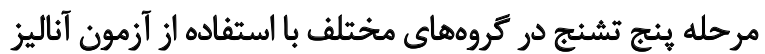

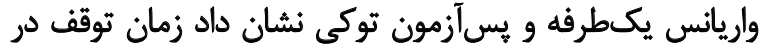

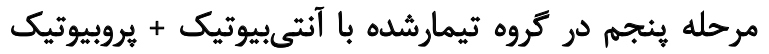

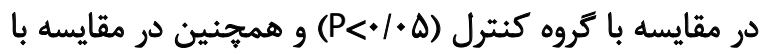

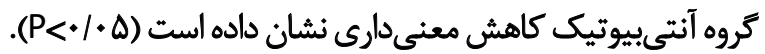

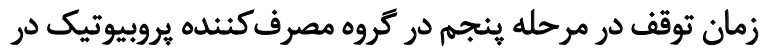

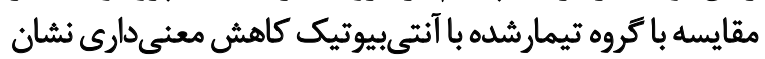

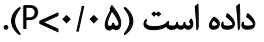

مقايسه ميانكين تعداد كلنى باكترى رشد بيداكرده در محيط MRS Agar

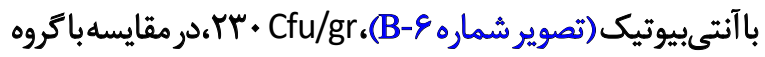

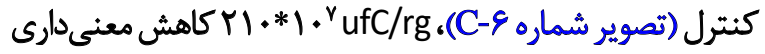

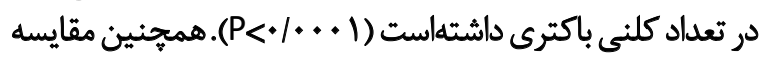

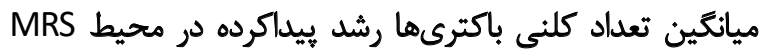
Agar

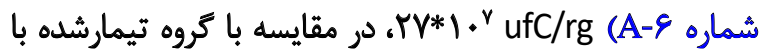

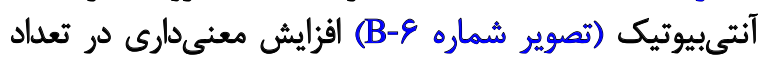

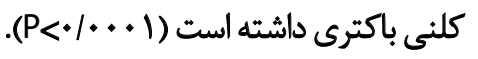

\section{بحث}

نتايج مطالعه حاضر نشان داد مصرف آنتىبيوتيكها موجب

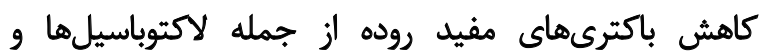

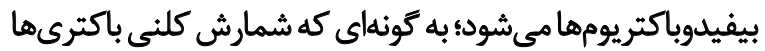

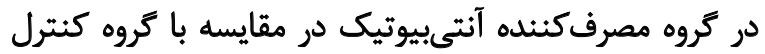

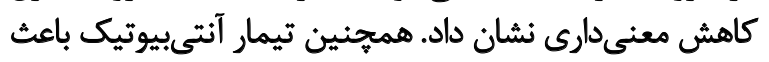

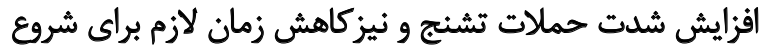

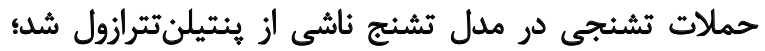

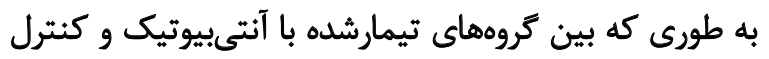

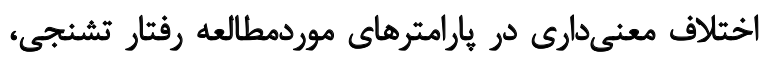




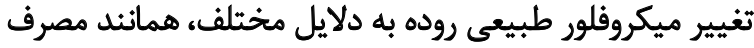

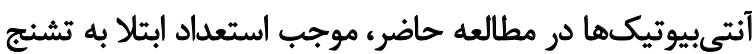

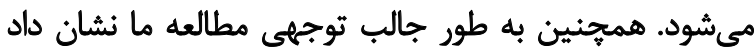

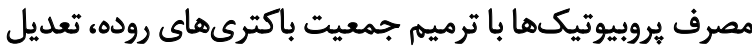

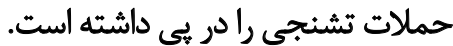

\section{تثيجلكَيرى}

با مطالعه شواهد موجود، به نظر مىرسد حذف فلور طبيعى

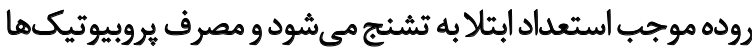

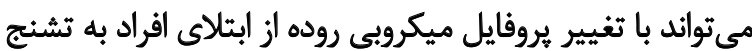

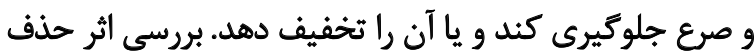

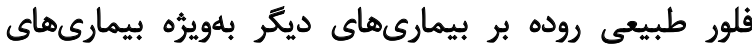

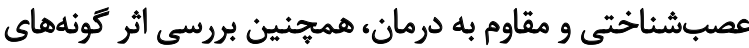

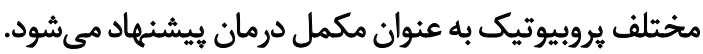

مالاحظات اخلاقي

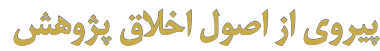

IR.ARAKMU.REC.1395.176 اين مطالعه با كد اخلاق توسط كميته اخلاق يثرهشى دانشكاه علوميزشكى اراك بات به

تصويب رسيده است.

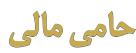

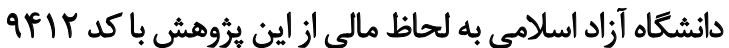

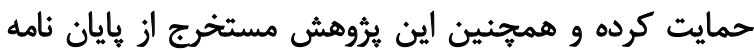

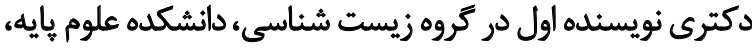
دانشكاه آزاد اسلامي واحد علوم و تحقيقات است.

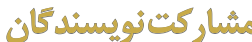

تمامى نويسندكان در نكارش اين مقاله مشاركت داشتهاند.

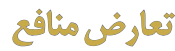

نويسندكان مقاله هيجَّونه تعارضى در منافع اعلام نكردند.

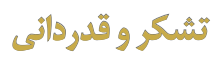

از دانشكاه آزاد اسلامى، واحد علوم و تحقيقات تهران به خاطر حمايت مالى تقدير و تشكر مى زعردد.

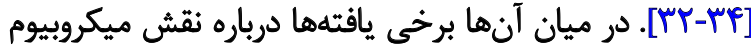

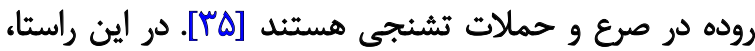

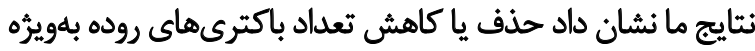

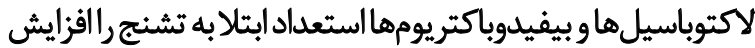

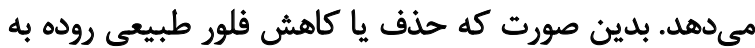

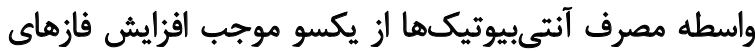

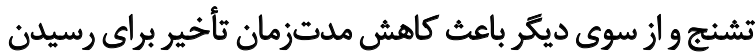

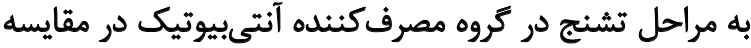

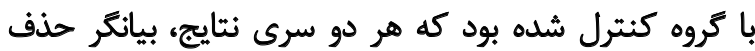

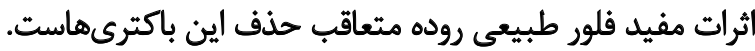

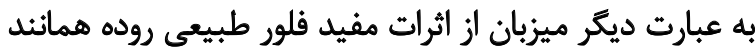

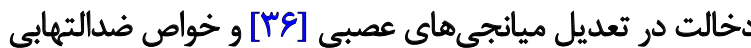

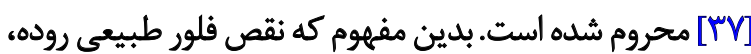
خود مي تواند دليلى بر ايجاد بيمارى يا يا تشديد آن باشد.

بررسىهاى قبلى نيز تغيير فلور طبيعى روده را در بيماران

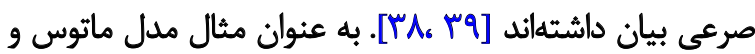

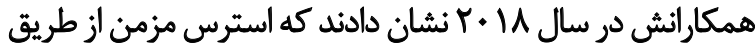

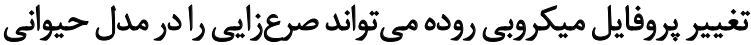

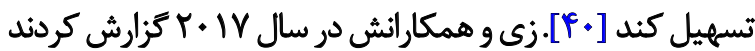

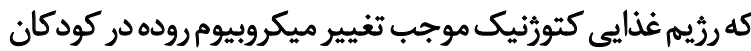

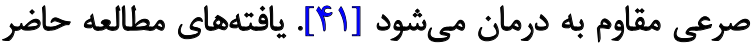

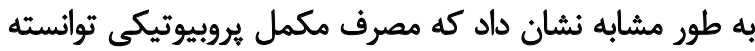

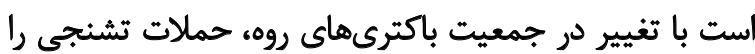

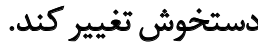

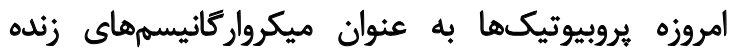

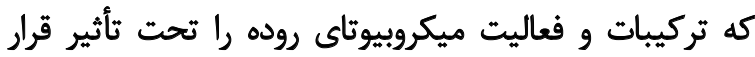
مى مهند، يذيرفته شدهاند [FT]

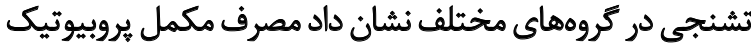

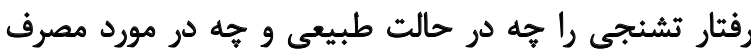

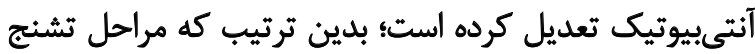

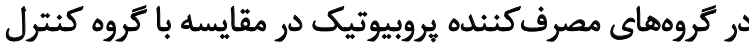

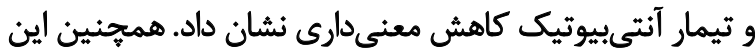

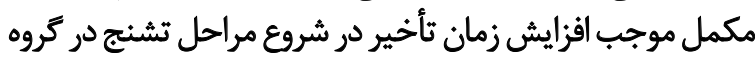

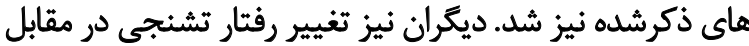

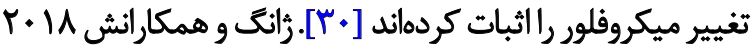

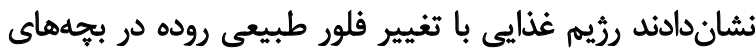

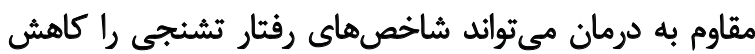

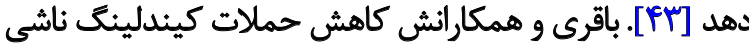

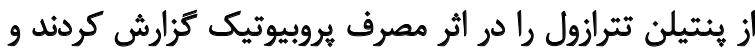

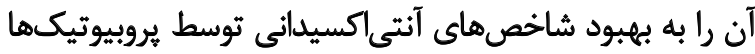

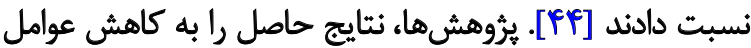

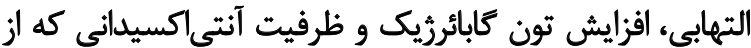

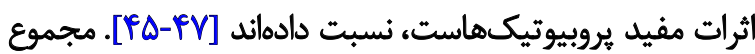

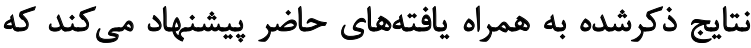




\section{References}

[1] Thurman DJ, Ettore B, Charles E, Berg AT, Buchhalter JR, Ding $D$, et al. Standards for epidemiologic studies and surveillance of epilepsy. Epilepsia. 2011; 52(Suppl. 7):2-26. [DOI:10.1111/j.15281167.2011.03121.x] [PMID]

[2] Holmes GL. The long-term effects of seizures on the developing brain: clinical and laboratory issues. Brain and Dev, 1991; 13(6):393-409. [DOI:10.1016/\$0387-7604(12)80037-4]

[3] Bradford H. Glutamate, GABA and epilepsy. Prog Neurobiol. 1995; 47(6):477-511. [DOI:10.1016/0301-0082(95)00030-5]

[4] Aguiar CCT, Almeida AB, Araújo PVP, de Abreu RNDC, Chaves EMC, Macêdo DS, et al. Oxidative stress and epilepsy: literature review. Oxidative medicine and cellular longevity, 2012; 2012:795259. [DOI:10.1155/2012/795259] [PMID] [PMCID]

[5] Vezzani A, Granata T. Brain inflammation in epilepsy: Experimental and clinical evidence. Epilepsia. 2005; 46(11):1724-43. [DOI:10.1111/j.15281167.2005.00298.x] [PMID]

[6] Galland L. The gut microbiome and the brain. J Med Food. 2014; 17(12):1261-72. [DOI:10.1089/jmf.2014.7000] [PMID] [PMCID]

[7] Lozupone CA, Stombaugh Jl, Gordon Jl, Jansson JK, Knight R. Diversity, stability and resilience of the human gut microbiota. Nature. 2012 489(7415):220-30. [DOI:10.1038/nature11550] [PMID] [PMCID]

[8] De Caro C, lannone LF, Citraro R, Striano P, De Sarro G, Constanti A, et al. Can we 'seize'the gut microbiota to treat epilepsy? Neurosci Biobehav Rev. 2019; 107:750-64. [DOI:10.1016/j.neubiorev.2019.10.002] [PMID]

[9] Cryan JF, O'mahony S. The microbiome-gut-brain axis: from bowel to behavior. Neurogastroenterol Motil. 2011; 23(3):187-92. [DOI:10.1111/ j.1365-2982.2010.01664.x] [PMID]

[10] Wu J, Zhang Y, Yang H, Rao Y, Miao J, Lu X. Intestinal microbiota as an alternative therapeutic target for epilepsy. Can J Infect Dis Med Microbiol. 2016; 2016:9032809. [DOI:10.1155/2016/9032809] [PMID] [PMCID]

[11] Delzenne NM, Neyrinck AM, Bäckhed F, Cani PD. Targeting gut microbiota in obesity: Effects of prebiotics and probiotics. Nat Rev Endocrinol. 2011; 7(11):639-46. [DOI:10.1038/nrendo.2011.126] [PMID]

[12] Dhakal R, Bajpai VK, and Baek KH. Production of GABA ( $\psi$-aminobutyric acid) by microorganisms: A review. Braz J Microbiol. 2012; 43(4):123041. [DOI:10.1590/S1517-83822012000400001] [PMID] [PMCID]

[13] Roselli M, Pieper R, Rogel-Gaillard C, de Vries H, Bailey M, Smidt H, et al. Immunomodulating effects of probiotics for microbiota modulation, gut health and disease resistance in pigs. Anim Feed Sci Technol. 2017; 233:104-19. [DOI:10.1016/j.anifeedsci.2017.07.011]

[14] Mazloom Z, Yousefinejad A, Dabbaghmanesh MH. Effect of probiotics on lipid profile, glycemic control, insulin action, oxidative stress, and inflammatory markers in patients with type 2 diabetes: A clinical trial. Iran J Med Sci. 2013; 38(1):38-43. [PMID] [PMCID]

[15] Cha BK, Jung SM, Choi CH, Song ID, Lee HW, Kim HJ, et al. The effect of a multispecies probiotic mixture on the symptoms and fecal microbiota in diarrhea-dominant irritable bowel syndrome: A randomized, doubleblind, placebo-controlled trial. J Clin Gastroenterol. 2012; 46(3):220-27. [DOI:10.1097/MCG.0b013e31823712b1] [PMID]

[16] Sansonetti PJ, Medzhitov R. Learning tolerance while fighting ignorance. Cell, 2009. 138(3):416-20. [DOl:10.1016/j. cell.2009.07.024] [PMID]
[17] Picard C, Fioramonti J, Francois A, Robinson T, Neant F, Matuchansky C Bifidobacteria as probiotic agents-physiological effects and clinical benefits. Aliment Pharmacol Ther. 2005; 22(6):495-12. [DOI:10.1111/j.13652036.2005.02615.x] [PMID]

[18] Riedel CU, Foata F, Philippe D, Adolfsson O, Eikmanns BJ, Blum S, et al. Anti-inflammatory effects of bifidobacteria by inhibition of LPS-induced NF-KB activation. World J Gastroenterol. 2006; 12(23):3729-35. [DOI:10.3748/wjg.v12.i23.3729] [PMID] [PMCID]

[19] Harmsen HJ, Wildeboer-Veloo AC, Raang GC, Wagendorp AA, Klijn $\mathrm{N}$, Bindels JG, et al. Analysis of intestinal flora development in breastfed and formula-fed infants by using molecular identification and detection methods. J Pediatr Gastroenterol Nutr. 2000; 30(1):61-7. [DOI:10.1097/00005176-200001000-00019] [PMID]

[20] Harmsen HJ, Raangs GC, He T, Degener JE, Welling GW. Extensive set of 165 rRNA-based probes for detection of bacteria in human feces. Appl Environ Microbiol. 2002; 68(6):2982-90. [DOI:10.1128/AEM.68.6.29822990.2002] [PMID] [PMCID]

[21] Dinan TG, Stilling, Roman M, Stanton C, Cryan JF. Collective unconscious: how gut microbes shape human behavior. J Psychiatr Res. 2015; 63:1-9. [DOI:10.1016/j.jpsychires.2015.02.021] [PMID]

[22] O'Mahony L, McCarthy j, Kelly P, Hurley G, Luo F, Chen K, et al. Lactobacillus and bifidobacterium in irritable bowel syndrome: Symptom responses and relationship to cytokine profiles. Gastroenterology. 2005 128:541-51. [DOI:10.1053/j.gastro.2004.11.050] [PMID]

[23] Cho I, Yamanishi S, Cox L, Methé BA, Zavadil J, Li K, et al. Antibiotics in early life alter the murine colonic microbiome and adiposity. Nature; 2012; 488(7413):621-6. [DOI:10.1038/nature11400] [PMID] [PMCID]

[24] Blaser MJ. Antibiotic use and its consequences for the normal microbiome. Science. 2016; 352(6285):544-5. [DOI:10.1126/science.aad9358] [PMID] [PMCID]

[25] Sullivan Å, Edlund C, Nord CE. Effect of antimicrobial agents on the ecological balance of human microflora. Lancet Infect Dis. 2001; 1(2):101-14. [DOI:10.1016/S1473-3099(01)00066-4] [PMID]

[26] Zhou J, Pillidge C, Gopal P, Gill HS. Antibiotic susceptibility profiles of new probiotic Lactobacillus and Bifidobacterium strains. Int J Food Microbiol. 2005; 98(2):211-7. [DOI:10.1016/j.ijfoodmicro.2004.05.011] [PMID]

[27] Plummer SF, Garaiova I, Sarvotham T, Cottrell SL, Scouiller SL, Weaver MA, et al. Effects of probiotics on the composition of the intestinal microbiota following antibiotic therapy. Int J Antimicrob Agents. 2005; 26(1):69-74. [DOI:10.1016/j.ijantimicag.2005.04.004] [PMID]

[28] Davoudi M, Shojaei A, Palizvan MR, Javan M, Mirnajafi-Zadeh J. Comparison between standard protocol and a novel window protocol for induction of pentylenetetrazol kindled seizures in the rat. Epilepsy Res. 2013; 106(1-2):54-63. [DOI:10.1016/j.eplepsyres.2013.03.016] [PMID]

[29] Narayanan R, Raghavan KT. Antibiotic susceptibility profile of lactic acid bacteria with probiotic potential isolated from humans.Biomed J Sci Tech Res. 2019; 17(4):12964-6. [DOI:10.26717/BJSTR.2019.17.003033]

[30] Ferrer M, Méndez-García C, Rojo D, Barbas C, Moya A. Antibiotic use and microbiome function. Biochem Pharmacol. 2017; 134:114-26. [DOI:10.1016/j.bcp.2016.09.007] [PMID]

[31] Allori C, Agüero G, de Ruiz Holgado AP, de Nader OM, Perdigon G. Gut mucosa morphology and microflora changes in malnourished mice after renutrition with milk and administration of Lactobacillus casei. J Food Prot. 2000; 63(1):83-90. [DOI:10.4315/0362-028X-63.1.83] [PMID] 
[32] Beilharz J, Kaakoush N, Maniam J, Morris MJ. Cafeteria diet and probiotic therapy: cross talk among memory, neuroplasticity, serotonin receptors and gut microbiota in the rat. Mol psychiatry. 2018; 23(2):35161. [DOI:10.1038/mp.2017.38] [PMID]

[33] Bercik P, Denou E, Collins J, Jackson W, Lu J, Jury J, et al. The intestinal microbiota affect central levels of brain-derived neurotropic factor and behavior in mice. Gastroenterology. 2011; 141(2):599-609. e3. [DOI:10.1053/j.gastro.2011.04.052] [PMID]

[34] Borre YE, O'Keeffe GW, Clarke G, Stanton C, Dinan TG, Cryan JF. Microbiota and neurodevelopmental windows: Implications for brain disorders. Trends Mol Med. 2014; 20(9):509-18. [DOI:10.1016/j.molmed.2014.05.002] [PMID]

[35] Lum GR, Olson CA, and Hsiao EY. Emerging roles for the intestinal microbiome in epilepsy. Neurobiol Dis. 2020; 135:104576. [DOI:10.1016/j. nbd.2019.104576] [PMID]

[36] Foster JA, Neufeld KAM. Gut-brain axis: How the microbiome influences anxiety and depression. Trends Neurosci. 2013; 36(5):305-12. [DOI:10.1016/j.tins.2013.01.005] [PMID]

[37] MacFabe DF, Cain NE, Boon F, Ossenkopp KP, Cain DP. Effects of the enteric bacterial metabolic product propionic acid on object-directed behavior, social behavior, cognition, and neuroinflammation in adolescent rats: Relevance to autism spectrum disorder. Behav Brain Res. 2011; 217(1):47-54. [DOI:10.1016/j.bbr.2010.10.005] [PMID]

[38] Lindefeldt M, Eng A, Darban H, Bjerkner A, Zetterström ZK, Allander $\mathrm{T}$, et al. The ketogenic diet influences taxonomic and functional composition of the gut microbiota in children with severe epilepsy. NPJ Biofilms Microbiomes. 2019; 5(1):1-13. [DOI:10.1038/s41522-018-0073-2] [PMID] [PMCID]

[39] Peng A, Qiu X, Lai W, Li W, Zhang L, Zhu X, et al. Altered composition of the gut microbiome in patients with drug-resistant epilepsy. Epilepsy Res. 2018; 147:102-7. [DOI:10.1016/j.eplepsyres.2018.09.013] [PMID]

[40] Medel-Matus JS, Shin D, Dorfman E, Sankar R, Mazarati A. Facilitation of kindling epileptogenesis by chronic stress may be mediated by intestinal microbiome. Epilepsia Open. 2018; 3(2):290-4. [DOI:10.1002/ epi4.12114] [PMID] [PMCID]

[41] Xie G, Zhou Q, Qiu CZ, Dai WK, Wang HP, Li YH, et al. Ketogenic diet poses a significant effect on imbalanced gut microbiota in infants with refractory epilepsy. World J Gastroenterol. 2017; 23(33):6164-71. [DOI:10.3748/wjg.v23.i33.6164] [PMID] [PMCID]

[42] Galdeano CM, de Moreno de LeBlanc A, Vinderola G, Bonet ME, Perdigón G. Proposed model: Mechanisms of immunomodulation induced by probiotic bacteria. Clin Vaccine Immunol. 2007; 14(5):485-92. [DOI:10.1128/CVI.00406-06] [PMID] [PMCID]

[43] Zhang Y, Zhou S, Zhou Y, Yu L, Zhang L, Wang Y. Altered gut microbiome composition in children with refractory epilepsy after ketogenic diet. Epilepsy Res. 2018; 145:163-8. [DOI:10.1016/j. eplepsyres.2018.06.015] [PMID]

[44] Bagheri S, Heydari A, Alinaghipour A, Salami M. Effect of probiotic supplementation on seizure activity and cognitive performance in PTZ-induced chemical kindling. Epilepsy Behav. 2019; 95:43-50. [DOI:10.1016/j.yebeh.2019.03.038] [PMID]

[45] Asemi Z, Zare Z, Shakeri H, Sabihi SS, Esmaillzadeh A. Effect of multispecies probiotic supplements on metabolic profiles, hs-CRP, and oxidative stress in patients with type 2 diabetes. Ann Nutr Metab. 2013; 63(12):1-9. [DOI:10.1159/000349922] [PMID]

[46] Bravo JA, Forsythe P, Chew MV, Escaravage E, Savignac HM, Dinan TG, et al. Ingestion of Lactobacillus strain regulates emotional behav- ior and central GABA receptor expression in a mouse via the vagus nerve. Proc Natl Acad Sci USA. 2011; 108(38):16050-5. [DOI:10.1073/ pnas.1102999108] [PMID] [PMCID]

[47] Cui HH, Chen CL, Wang JD, Yang YJ, Cun Y, Wu JB, et al. Effects of probiotic on intestinal mucosa of patients with ulcerative colitis. World J Gastroenterol. 2004; 10(10):1521-5. [DOI:10.3748/wjg.v10.i10.1521] [PMID] [PMCID] 
This Page Intentionally Left Blank 